\title{
Casimir effect in an axially symmetric spacetime with unparticles
}

\author{
V. B. Bezerra ${ }^{1, a}$, C. R. Muniz ${ }^{2, b}$, H. S. Vieira ${ }^{3, c}$ \\ ${ }^{1}$ Departamento de Física, CCEN, Universidade Federal da Paraíba, Caixa Postal 5008, João Pessoa, PB, Brazil \\ ${ }^{2}$ Grupo de Física Teórica (GFT), Universidade Estadual do Ceará, Faculdade de Educação, Ciências e Letras de Iguatu, Av. Dário Rabelo, s/n, \\ Iguatu, CE CEP 63500-000, Brazil \\ ${ }^{3}$ Instituto de Física de São Carlos, Universidade de São Paulo, Caixa Postal 369, São Carlos, São Paulo CEP 13560-970, Brazil
}

Received: 8 August 2019 / Accepted: 9 October 2019 / Published online: 26 October 2019

(C) The Author(s) 2019

\begin{abstract}
We investigate the Casimir effect of the massless scalar field in a cavity formed by ideal parallel plates in the spacetime generated by a rotating axially symmetric distribution of vector or scalar (tensor) unparticles, around which the plates orbit. The presence of the unparticles is incorporated to the background by means of a correction to the Kerr solution of the Einstein equations, in which the characteristic length and the scale dimension associated to the unparticle theory are taken into account. We show that the Casimir energy density depends also on these parameters. The analysis of the "ungravity" limit for the Casimir energy density, in which the characteristic length is very large in comparison to the horizon radius, is made, too. At zero temperature, we show that such a limit implies the instability of the system, since the Casimir energy density becomes an imaginary quantity. The general result is compared to the current terrestrial experiments of the Casimir effect. Thermal corrections also are investigated and the ungravity limit again examined, with the aforementioned instability disappearing at high temperatures.
\end{abstract}

\section{Introduction}

The Standard Model (SM) of particles and fields seems to have reached the limit of its extraordinary predictive capacity. The 27-km-perimeter Large Hadron Collider (LHC) has so far successfully confirmed this model, with relatively few surprises revealed since its first operations started ten years ago. There are still a plethora of data to be processed and analyzed, which will take some time. Irrespective of this, one must search for more information through alternative experiments which probe other phenomena, in order to test

\footnotetext{
a e-mail: valdir@ fisica.ufpb.br

be-mail: celio.muniz@uece.br

c e-mail: horacio.santana.vieira@hotmail.com
}

theories which go beyond the SM, since there are several opened questions that this model does not answer.

In addition to leaving out a consistent quantum description of gravity, other unanswered questions by the SM are the dark matter origin and why matter survived annihilation with antimatter in different stages after the Big Bang [1,2]. One of the proposals to explain the former involves the existence of new particles out of the SM, which includes WIMPs (Weakly Interacting Massive Particles), axion-like particles and sterile neutrinos [3]. On the other hand, extensions of the SM based on Supersymmetry may explain the asymmetry matter-antimatter by proposing the existence of new massive particles and interactions which break the time-reversal symmetry, endowing, in addition, common charged leptons with an electric dipole moment aligned with the particle's spin [4].

Just as the breakdown of some symmetries of the SM implies the existence of new particles, the symmetry previously restricted to the massless sector of the model-the conformal invariance - may be extended to a new category of microscopic objects termed unparticles, proposed some time ago by Georgi $[5,6]$. These entities also could account for both the dark matter nature and baryon asymmetry $[7,8]$. They have undefined mass or continuous values for it [9] depending on the energy scale at which one detects them, i.e., the usual energy-momentum dispersion relation for a free particle is not longer valid, hence the name unparticle. An usual particle only owns scale invariance if it is massless. The unparticles, even endowed with mass, though indefinite, enjoys that property. Beside this, the scale dimension of the fields in the action can be fractionary, which leads to the representation of non-integral numbers of massless particles.

The unparticle proposal was inspired in the older theory of Bank-Zacks [10], where a conformal invariant high energy sector near a critical (fixed) point is possible, around which there would be fields of unknown nature weakly coupled to those ones of the SM. However, the very weak magnitude 
of the couplings would impose serious restrictions to the detection of the unparticles. Despite this fact, some events in the domain of high energies could indicate their presence [11-13] as well as in Astrophysics and Cosmology [14-20], and also in low energies phenomena [21,22], including the Casimir Effect [23]. In this case, the fractionary character of the scale dimension of the fields reflects in the dimensionality of the plates: It is non-integral, presenting a fractal nature, therefore.

The Casimir effect was discovered in 1948 as the attractive force arising between two parallel and uncharged metallic plates placed in vacuum, which results from the modification of the zero point oscillations of the electromagnetic field induced by the material boundaries [24-27]. Nowadays there are no doubt about the existence of this effect confirmed by many accurate experiments which have been performed during the last twenty years. Generically, the phenomenon also occurs when the vacuum of an arbitrary quantum field is disturbed by the presence of boundaries with different shapes and made of different materials, usually revealing itself through a force that arises on or between such boundaries [28]. The disturbance of the quantum vacuum can arise also on empty spaces with nontrivial topology $[29,30]$. This phenomenon can still be associated to a quantum field with arbitrary spin describing baryonic or even exotic matter [31]. The progressive increment in the precision of the Casimir effect measurements show that they tend to be a relevant source of tests for both high energy physics and modified theories of gravity [32-35].

In this paper, we will investigate the Casimir effect associated to a massless scalar field in a cavity formed by two ideal parallel plates, which are placed in the spacetime generated by a rotating axially symmetric gravitational source based on a distribution of vector or scalar (tensor) unparticles. This work extends the one that studied the phenomenon in the flat spacetime considering only scalar (tensor) unparticles [23]. Here, the computation of the Casimir energy density will follow the approach contained in [36] according to which it is made a coordinate transformation that will allow us to use a Cartesian coordinate system associated to the rectangular cavity. The presence of the unparticles is incorporated to the background by taking the axially symmetric solutions of the Einstein field equations obtained in [20]. The analysis of the "ungravity" limit for the Casimir energy density, in which the characteristic length of the theory is very large in comparison to the horizon radius $[16,17]$, will be made, too. The obtained result will be compared to the current experiments about the Casimir effect. Thermal corrections also will be investigated and the aforementioned limit again examined.

This paper is organised as follows: In Sect. 2 we review the unparticle features in the gravitational scenario. In Sect. 3 we compute the Casimir energy density in the parallel plates configuration. In Sect. 3.1 we analyse the thermal corrections and, finally, in Sect. 4 we close the paper with the conclusions.

\section{Unparticle static black holes}

In this section we briefly review the solution for the scalar (tensor) and vector unparticle static black holes found in Refs. $[16,17]$. The action is given by $S=S_{M}+S_{U}$, where $S_{M}$ is the matter action

$$
\begin{aligned}
S_{M} & \equiv-\int d^{4} x \sqrt{g} \rho(x) g_{\mu \nu} u^{\mu} u^{\nu}, \\
\rho(x) & \equiv \frac{M}{\sqrt{g}} \int d \tau \delta(x-x(\tau))
\end{aligned}
$$

and $S_{U}$ is the sum of the Einstein-Hilbert action and a correction due to the unparticles, given by

$$
\begin{aligned}
S_{U}= & \frac{1}{2 \kappa^{2}} \int d^{4} x \sqrt{g}\left[1+\frac{A_{d_{U}}}{\left(2 d_{U}-1\right) \sin \left(\pi d_{U}\right)} \frac{\kappa_{*}^{2}}{\kappa^{2}}\right. \\
& \left.\left(\frac{-D^{2}}{\Lambda_{U}^{2}}\right)^{1-d_{U}}\right]^{-1} R .
\end{aligned}
$$

In the above expression $D^{2}$ stands for the d'Alambertian and $d_{U}$ is the scale dimension. The parameters $A_{d_{U}}$ and $\kappa_{*}$ are

$A_{d_{U}} \equiv \frac{16 \pi^{5 / 2}}{(2 \pi)^{2 d_{U}}} \frac{\Gamma\left(d_{U}+1 / 2\right)}{\Gamma\left(d_{U}-1\right) \Gamma\left(2 d_{U}\right)}$

and

$\kappa_{*} \equiv \frac{1}{\Lambda_{U}}\left(\frac{\Lambda_{U}}{M_{U}}\right)^{d_{U V}}$.

The strength of the coupling constant is determined by the mass scale $M_{U}$ which replaces the Planck mass. The parameter $\Lambda_{U}$ is the energy scale at which occurs the dimensional transmutation associated to the presence of the unparticles. By assuming a static source, the analytical solution for the spherically symmetric geometry is given by $[16,17]$

$g_{r r}^{-1}=-g_{00}=1-\frac{2 M}{r}\left[1 \pm\left(\frac{R_{s, v}}{r}\right)^{2 d_{U}-2}\right]$

where

$$
\begin{aligned}
R_{s, v} & =\left[\Gamma_{U} \frac{M_{P l .}^{2} \kappa_{s, v}^{2}}{\pi^{2 d_{U}-1}} \Lambda_{U}^{2-2 d_{U}}\right]^{\frac{1}{2 d_{U}-2}} ; \\
\Gamma_{U} & =\frac{\Gamma\left(d_{U}-1 / 2\right) \Gamma\left(d_{U}+1 / 2\right)}{\Gamma\left(2 d_{U}\right)},
\end{aligned}
$$


$k_{s, v}$ are the coupling constants of the $s, v$ unparticles with gravity and $M_{P l}$ is the Planck mass. The plus signal is taken for the scalar (tensor) unparticle case $\left(R_{S}\right)$ and the minus one for the vector case $\left(R_{v}\right)$. These solutions have horizon curves defined by $g_{r r}^{-1}=0$, and thus we get

$M=\frac{r_{H}}{2} \frac{1}{1 \pm\left(R_{s, v} / r_{H}\right)^{2 d_{U}-2}}$.

In Refs. [16,17] two regimes are considered, namely, the gravity dominated (GD) regime, which corresponds to $R_{s, v} \ll r_{H}$, and the ungravity dominated (UGD) regime, for which $r_{H} \ll R_{s, v}$. In the GD regime, for both cases, the mass parameter reduces to the standard $M=r_{H} / 2$. However, in the UGD regime the mass is given by

$M \approx \pm \frac{1}{2} \frac{r_{H}^{2 d_{U}-1}}{R_{s, v}^{2 d_{U}-2}}$.

Therefore, for the vector case, the mass parameter becomes negative in the UGD regime. In fact for the entire region $r_{H}<R_{v}$ this parameter is negative. This fact lead the authors of Ref. [16] to ignore this region. However, it is a known fact that a negative mass parameter indicates black hole solution with nontrivial topology [37]. This suggests that beyond the fractalization of the horizon surface, the vector unparticle can in fact contribute to changes in the black hole topology. Since the UGD regime must be contained in a full quantum gravity theory, it is expected that quantum fluctuations in the geometry should enhance quantum fluctuations in the topology [38].

\subsection{Unparticles and quintessence in Kerr-like spacetimes}

The static solution for an unparticle black hole is something similar to the quintessence one [40], in which

$$
\begin{aligned}
\left(g_{r r}^{q}\right)^{-1}= & -g_{00}^{q}=1-\frac{2 M}{r}-\frac{\alpha}{r^{3 \omega_{q}+1}} \Leftrightarrow\left(g_{r r}^{u}\right)^{-1}=-g_{00}^{u}=1 \\
& -\frac{2 M}{r}\left[1 \pm\left(\frac{R_{s, v}}{r}\right)^{2 d_{U}-2}\right]
\end{aligned}
$$

where $\alpha \geq 0$ and $\omega$ is constrained, by cosmological evidences associated with the accelerated expansion of the Universe, to $-1 \leq \omega \leq-1 / 3$ [40]. In fact the mapping between them comes from the identification

$3 \omega+1=2 d_{U}-1 ; \alpha= \pm 2 M R_{s, v}^{2 d_{U}-2}$.

This suggests that unparticles could be a source of quintessential matter for astrophysical objects. Since $\omega$ has a range of permitted values, the above relationship between $\omega$ and $d_{U}$ implies that $-1 / 2 \leq d_{U} \leq 1 / 2$. However, this is not allowed since, by unitarity reasons, $d_{U} \geq 1$ for scalar (tensor) unparticles and $d_{U} \geq 3$ for the vector ones [39]. Therefore, the static black hole quintessence can not be described by unparticles. Despite of this, the formal similarity of the above solutions will guide us to generalize the unparticle black hole to the case with rotation. We can construct the solution corresponding to the rotating unparticle black hole. The standard procedure is to use the Newman-Janis algorithm. However we can take a shortcut since, as said before, our static solution is formally identical to the one with quintessence found in Ref. [40]. By using the referred algorithm with the choice of different complexifications for the radial coordinate, solutions for the rotating black hole surrounded by quintessence were independently found by Ghosh [41] and Toshmatov et al. [42]. The solutions in the Boyer-Lindquist coordinates are given by

$$
\begin{aligned}
d s^{2}= & \left(1-\frac{2 M r}{\Sigma}\right) d t^{2}-\frac{\Sigma}{\tilde{\Delta}_{1,2}} d r^{2}+\frac{4 M a r}{\Sigma} \sin ^{2} \theta d t d \phi \\
& -\Sigma d \theta^{2}-\frac{\tilde{A}_{1,2}}{\Sigma} d \phi^{2}
\end{aligned}
$$

where $\Sigma=r^{2}+a^{2} \cos ^{2} \theta$ and

$\tilde{\Delta}_{1,2}=r^{2}+a^{2}-2 M r-\alpha F_{1,2}^{1-3 \omega}$.

and

$\tilde{A}_{1,2}=\left(r^{2}+a^{2}\right)^{2}-a^{2} \tilde{\Delta}_{1,2} \sin ^{2} \theta$

The subscripts 1 (2) refers to the Ghosh (Toshmatov et al.) solution and the functions $F_{1,2}$ are given by

$F_{1}=\Sigma^{\frac{1}{2}} ; F_{2}=r$.

Now we apply our mapping (9) in order to obtain the solution for the rotating unparticle black holes, which is given by

$\tilde{\Delta}_{U}=r^{2}+a^{2}-2 M r \pm(2 M) R_{s v}^{2 d_{U}-2} F_{1,2}^{3-2 d_{U}}$.

From this metric we can obtain the mass parameter of the black holes, which is obtained by imposing that $\tilde{\Delta}=0$ at the horizon, $r_{H}$. Thus, we obtain

$2 M=\frac{r_{H}^{2}+a^{2}}{r_{H} \pm R_{s v}^{2 d_{U}-2} F_{1,2}^{3-2 d_{U}}}$.

Therefore, the mass parameter depends strongly on what solutions will be taken into account, if that obtained by Ghosh [41] or the one obtained by Toshmatov et al. [42]. 


\section{Casimir effect}

In this section, using the metric given by Eq. (10), we will obtain the vacuum energy of a scalar field enclosed in a rectangular cavity nearby the black hole under consideration. The cavity we will consider is the region between two metallic plates localized in a locally co-moving reference frame which describes a circular orbit around the unparticle black hole equator, with angular velocity $\Omega$. Suppose that a frame of Cartesian coordinates $(x, y, z)$ is centered on one of these plates such that the $z$ axis is tangential to the path of the circular orbit $[36,43]$. In this case, the spherical coordinates centered on the source and the Cartesian axes of the orbiting system are related by $d y=d r, d z=r d \phi^{\prime}$, and $d x=-r d \theta$, where $\phi^{\prime}=\phi-\Omega t$. Therefore, in the Cartesian frame, the metric given by Eq. (10) can be written as

$$
\begin{aligned}
d s^{2}= & \tilde{C}^{-2}(\Omega) d t^{2}-\frac{\tilde{A}_{1,2}}{r \Sigma} \sin ^{2} \theta\left(\tilde{\omega}_{d}-\Omega\right) d t d z-\frac{\Sigma}{\tilde{\Delta}_{1,2}} d y^{2} \\
& -\frac{\Sigma}{r^{2}} d x^{2}-\frac{\tilde{A}_{1,2}}{r^{2} \Sigma} \sin ^{2} \theta d z^{2}
\end{aligned}
$$

where

$\tilde{\omega}_{d}=-\frac{g_{t \phi}}{g_{\phi \phi}}=\frac{2 M a r}{\tilde{A}_{1,2}}$

is the spacetime dragging angular velocity and

$$
\tilde{C}^{-2}(\Omega)=\frac{\Sigma \tilde{\Delta}}{\tilde{A}_{1,2}}\left[1-\frac{\tilde{A}_{1,2}^{2}}{\tilde{\Delta}_{1,2} \Sigma^{2}} \sin ^{2} \theta\left(\Omega-\tilde{\omega}_{d}\right)^{2}\right] .
$$

The Klein-Gordon equation which describes a scalar field minimally coupled with gravity is

$$
\frac{1}{\sqrt{-g}} \partial_{\mu}\left(\sqrt{-g} g^{\mu \nu} \partial_{\nu}\right) \Phi(t, x, y, z)=0 .
$$

Using Eq. (19) and the metric given by Eq. (16), it is possible to determine the quasinormal modes and the vacuum energy between the parallel plates, from which one obtains the renormalized vacuum energy by following the same approach described in [36]. It is given by the following expression

$$
\begin{aligned}
& \left\langle\epsilon_{\text {vac }}\right\rangle_{1,2}^{(\text {ren })}= \\
& -\frac{\pi^{2}}{1440 L_{p}^{4}} \sqrt{\frac{\Sigma}{r^{2}}}\left[1-\frac{\tilde{A}_{1,2}^{2}}{\tilde{\Delta}_{1,2} \Sigma^{2}} \sin ^{2} \theta\left(\Omega-\tilde{\omega}_{d}\right)^{2}\right]^{\frac{1}{2}},
\end{aligned}
$$

in which the proper length $L_{p}$ is defined by

$$
L_{p}=\tilde{C} \frac{\sin \theta \sqrt{\tilde{\Delta}}}{r} L
$$

In the ungravity regime, in which $R_{S, v} / r_{H} \gg 1$, one can show that the system is unstable, since the Casimir energy becomes an imaginary quantity, given by

$\left\langle\epsilon_{\text {vac }}\right\rangle_{\text {un }}^{(\text {ren })} \approx-i \frac{\pi^{2}}{1440 L_{p}^{4}} \sqrt{\frac{r_{H}^{2}+a^{2}}{r^{2} \Sigma}} a^{2} \Omega \sin ^{3} \theta$,

where we considered Eq. (15). This result is the same for both the scalar or vector unparticles, as well as for both the prescriptions given by Ghosh [41] and Toshmatov et al. [42]. It is worth mention, as we will see in details, that in the thermal Casimir effect there is no instability. On the other hand, in the opposite regime, $R_{s, v} / r_{H} \ll 1$, we reach the result given in [36].

We may infer bounds on the characteristic distance $R_{S}$ of the scalar unparticle gravity from the precision of the measurements of the Casimir effect performed on Earth surface, with $r=6.3 \times 10^{6} \mathrm{~m}$. The plates are considered orbiting the Earth in the same direction and at a rate of its daily rotation ( $\Omega=2 \pi / 86,400 \mathrm{~s}^{-1}$ ). Such bounds on $R_{S}$ can be computed from Eq. (20) as a function of the relative error $\delta_{\epsilon}$ in those measurements and they are given by

$R_{S} \gtrsim r\left[\frac{r^{3} \Omega^{2}}{G M \delta_{\epsilon}\left(\delta_{\epsilon}+2\right)}\right]^{\frac{1}{2\left(d_{U}-1\right)}}$,

where we neglected terms which depend on the square of the rotation parameter, $a^{2}$, and up. The reason to assume this is the fact that it is very tiny for Earth. The shadowed region depicted in Fig. 1 shows us the parameter space $\left(R_{S}, d_{U}\right)$ of the scalar ungravity by considering the current accuracy of the Casimir effect measurements, around $1 \%$. Notice that the scale dimension should not differ much from unity since such a fact would imply the improbable occurrence of the ungravity regime.

On the other hand, based on Eq. (5), we can find the upper bound on the energy scale of the unparticles, $\Lambda_{U}$, as a function of the scale dimension, $d_{U}$. The graph in Fig. 2 depicts this, where we have taken the coupling constant as being $\kappa_{s}=1$.

It is interesting to compare this result with that one given in [23], which considered the Casimir effect of scalar unparticles in the Minkowsky spacetime, with a relative error of the current measurements as being $\delta_{\epsilon}=30 \%$ and without dependence on the coupling constant. We obtain for the ungravity Casimir effect a very stronger bound.

\subsection{Thermal corrections to the Casimir energy}

Now, let us consider the system immersed in a thermal bath. The Helmholtz free energy calculated in a finite volume $V$ is given by [28] 


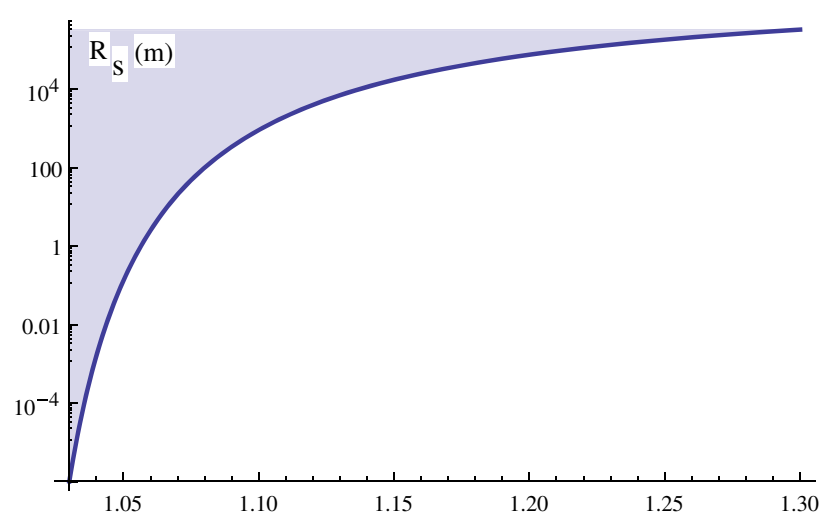

Fig. 1 Lower bound on $R_{S}$, the characteristic length of the scalar unparticle sector, in meters, as a function of the scale dimension, $d_{U}$, where we considered the relative error in actual measurements of the Casimir effect in the Earth gravitational field, as being of the order of $\delta_{\epsilon}=1 \%$. The shadowed region embraces the possible values of $d_{U}$ and $R_{S}$

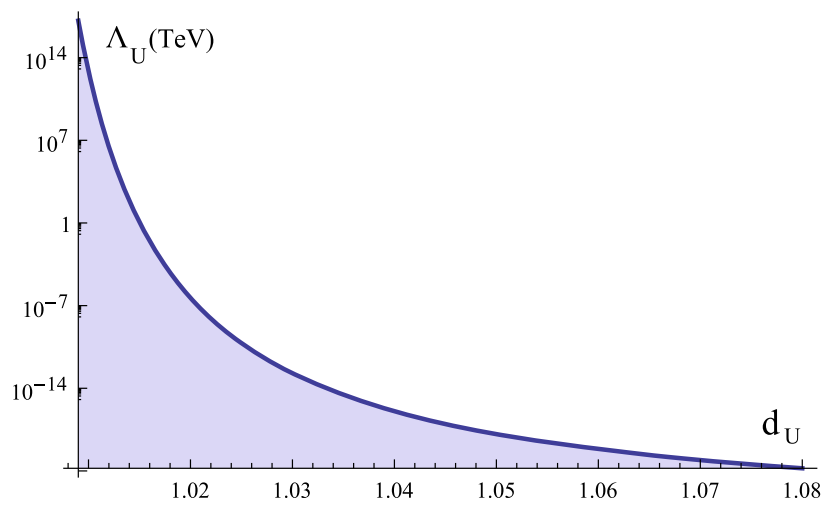

Fig. 2 Upper bound on $\Lambda_{U}$, the energy scale of scalar unparticles, as a function of the scale dimension, $d_{U}$, where we have taken the coupling constant $\kappa_{s}=1$ and the other parameters as the ones previously given

$\tilde{F}_{0}=E_{0}^{(r e n)}+\Delta_{T} F_{0}-\mathcal{O}\left(T^{2}\right)-\mathcal{O}\left(T^{3}\right)-V f_{b b}$

where $E_{0}^{(r e n)}=V_{p}\left\langle\epsilon_{v a c}\right\rangle^{(r e n)}$ is the vacuum energy and

$\Delta_{T} F_{0}=A k_{B} T \iint \frac{d^{2} \mathbf{k}}{(2 \pi)^{2}} \sum_{n} \log \left(1-e^{-\frac{\omega_{n}}{k_{B} T}}\right)$,

with $A$ being the area between the plates and $k_{B}$ is the Boltzmann constant. The term $f_{b b}$ is calculated using the expression

$f_{b b}=k_{B} T \iiint \frac{d^{3} \mathbf{k}}{(2 \pi)^{3}} \log \left(1-e^{-\frac{\omega(k)}{k_{B} T}}\right)$,

where $k$ is the modulus of the wave vector $\mathbf{k}$. The terms $\mathcal{O}\left(T^{2}\right)$ and $\mathcal{O}\left(T^{3}\right)$ are obtained by expanding the free energy. Defining the Helmholtz energy density by $\tilde{f}_{0}=\frac{\tilde{F}_{0}}{V_{p}}$ and calculating the internal energy density through the expression
$u_{0}(T)^{(r e n)}=-T^{2} \frac{\partial}{\partial T}\left(\frac{\tilde{f}_{0}}{T}\right)$

we find that the thermal corrections to the Casimir energy density are given by

$$
\begin{gathered}
u_{0}(T)^{(r e n)}=-\frac{\tilde{C}^{3}(\Omega, \theta) \pi^{2}\left(k_{B} T\right)^{4}}{30} \\
+\sum_{n, s=1}^{\infty}\left[\frac{n^{2} \pi\left(k_{B} T\right)}{2 s L_{p}^{3}}+\frac{n \tilde{C}(\Omega, \theta)\left(k_{B} T^{2}\right)}{s^{2} L_{p}^{2}}\right. \\
\left.+\frac{\tilde{C}(\Omega, \theta)\left(k_{B} T\right)^{3}}{s^{3} \pi L_{p}}\right] e^{-\frac{n s \pi}{k_{B} T \tilde{C}(\Omega, \theta) L_{p}}} .
\end{gathered}
$$

In the high temperature limit, the internal energy density is dominated by the black body subtraction $\left(T^{4}\right)$ term. A term proportional to $T^{3} / L_{P}$ was discarded since the thermal internal energy does not depend on $L_{P}$ and then there is no physical effects to consider. It is interesting to notice that in the ungravity regime, the dominant term of the thermal corrections to the Casimir energy, in the high temperature limit, is given by

$u_{0}(T)_{1,2}^{(r e n)} \approx-\frac{\Sigma^{3 / 2} \pi^{2}\left(k_{B} T\right)^{4}}{30 a^{3} \Omega^{3} \sin ^{3} \theta}$,

without any change of signal independent of the unparticle to be a scalar or vector, i.e., the Casimir thermal force between the plates will always be repulsive, and independent also of the prescriptions given by Ghosh [41] or Toshmatov et al. [42]. Notice that in the ungravity regime the thermal Casimir energy density at high temperatures is a real quantity, and therefore there is no longer instability in the system.

In the low temperature limit, the subdominant term of the thermal Casimir energy density, obtained by excluding the black body contribution, in the ungravity regime will be given by

$u_{0}(T)_{1,2}^{(r e n)} \approx-\left(\frac{\pi k_{B} T}{2 L_{p}^{3}}\right) \exp \left(\frac{\Sigma^{1 / 2}}{k_{B} T a \Omega \sin \theta L_{p}}\right)$,

for scalar (tensor) or vector unparticle black hole, irrespective of the solution considered, if the one obtained by Ghosh or the other obtained by Toshmatov et al.. Once more, we have obtained a real quantity, but in that regime the system remains unstable, due to the contribution of the Casimir energy density at zero temperature.

\section{Concluding remarks}

We have studied the contribution to the Casimir effect by a massless scalar field in a cavity formed by ideal parallel 
plates orbiting a rotating distribution of vector or scalar (tensor) unparticles, according to the Georgi formalism [5]. The presence of these entities was considered in an axially symmetric Kerr-like solution of the Einstein equations obtained in [20]. We taken into account the different technics of obtaining rotating solutions via Ghosh [41] or Toshmatov et al. [42] prescriptions, which depend on the characteristic length, $R_{s, v}$, and on the scale dimension, $d_{U}$, both the parameters associated to the unparticle theory. It was made a transformation in the Kerr-like metric in order to associate a Cartesian coordinate system to the rectangular cavity. Thus, the computation of the vacuum energy, including its regularization, was done by following Sorge approach [36]. The obtained results show that the Casimir energy density depends on those unparticle parameters.

The analysis of the limit in which the characteristic length is very large in comparison to the horizon radiusthe ungravity regime-was made, and we concluded that, at zero temperature, the system is unstable since the Casimir energy density becomes an imaginary quantity. The computed Casimir energy density was then compared to the result of the actual experiments realized with the Casimir effect, and from this we have graphically pointed out a set of allowed magnitudes for $R_{S}$ and $d_{U}$, as well as for $\Lambda_{U}$ and $d_{U}$, in the parameter space. In fact, the current measurements of the Casimir effect point to a scale dimension slightly different from unity, since otherwise we would live in a world in which predominates the ungravity regime, which does not seem to be the case. Furthermore, the proposed case of the plates on the Earth offers stronger limits on unparticles than the one registered in the Minkowsky spacetime according to [23]. Finally, thermal corrections to the Casimir energy density were investigated and the ungravity limit again examined, with the aforementioned instability disappearing at high temperatures.

Acknowledgements The authors thank CNPq and FUNCAP for their partial support under the grant PRONEM PNE-0112-00085.01.00/16. H.S.V. is funded by the Coordenação de Aperfeiçoamento de Pessoal de Nível Superior (CAPES)-Finance Code 001.

Data Availability Statement This manuscript has no associated data or the data will not be deposited. [Authors' comment: The present article contains itself all the informations and data necessary for its full understanding.]

Open Access This article is distributed under the terms of the Creative Commons Attribution 4.0 International License (http://creativecomm ons.org/licenses/by/4.0/), which permits unrestricted use, distribution, and reproduction in any medium, provided you give appropriate credit to the original author(s) and the source, provide a link to the Creative Commons license, and indicate if changes were made.

Funded by SCOAP ${ }^{3}$.

\section{References}

1. D. Kazakov, Beyond the Standard Model' 17. arXiv:1807.00148 [hep-ph]

2. V.P. Nair, Concepts in particle physics. https://doi.org/10.1142/ 10640

3. G. Bertone, T. Tait, M.P, A new era in the search for dark matter. Nature 562, 7725(51 ) (2018). https://doi.org/10.1038/ s41586-018-0542-z

4. V. Andreev et al. [ACME Collaboration], Improved limit on the electric dipole moment of the electron. Nature 562(7727), 355 (2018). https://doi.org/10.1038/s41586-018-0599-8

5. H. Georgi, Unparticle physics. Phys. Rev. Lett. 98, 221601 (2007). https://doi.org/10.1103/PhysRevLett.98.221601

6. H. Georgi, Another odd thing about unparticle physics. Phys. Lett. B 650, 275 (2007). https://doi.org/10.1016/j.physletb.2007.05.037

7. X.G. He, S. Pakvasa, Unparticle induced baryon number violating nucleon decays. Phys. Lett. B 662, 259 (2008). https://doi.org/10. 1016/j.physletb.2008.03.025

8. M.A. Deliyergiyev, Recent progress in search for dark sector signatures. Open Phys 14(1), 281 (2016). https://doi.org/10.1515/ phys-2016-0034

9. N.V. Krasnikov, Unparticle as a field with continuously distributed mass. Int. J. Mod. Phys. A 22, 5117 (2007). https://doi.org/10. 1142/S0217751X07037342

10. T. Banks, A. Zaks, On the phase structure of vector-like gauge theories with massless fermions. Nucl. Phys. B 196, 189 (1982). https://doi.org/10.1016/0550-3213(82)90035-9

11. D. Van Soa, B.T.H. Giang, The effect of the scalar unparticle on the production of Higg-radion at high energy colliders. Nucl. Phys. B 936, 1 (2018). https://doi.org/10.1016/j.nuclphysb.2018.09.003

12. S .T .L. Anh, P .T .H. Trang, T .Q. Trang, H .H. Bang, Unparticle effects on axion-like particles production in $\mathrm{e}^{+} \mathrm{e}$ collisions. Int. J. Theor. Phys 57(7), 2015 (2018). https://doi.org/10.1007/ s10773-018-3727-1

13. H. Bagheri, M. Ettefaghi, R. Moazzemi, On the difference of timeintegrated $C P$ asymmetries in $D^{0} \rightarrow K^{+} K^{-}$and $D^{0} \rightarrow \pi^{+} \pi^{-}$ decays: unparticle physics contribution. Phys. Lett. B 771, 309 (2017). https://doi.org/10.1016/j.physletb.2017.05.046

14. J.R. Mureika, Unparticle-enhanced black holes at the LHC. Phys. Lett. B 660, 561 (2008). https://doi.org/10.1016/j.physletb.2008. 01.050

15. J. McDonald, Cosmological constraints on unparticles. JCAP 0903 , 019 (2009). https://doi.org/10.1088/1475-7516/2009/03/019

16. J.R. Mureika, E. Spallucci, Vector unparticle enhanced black holes: exact solutions and thermodynamics. Phys. Lett. B 693, 129 (2010). https://doi.org/10.1016/j.physletb.2010.08.025

17. P. Gaete, J.A. Helayel-Neto, E. Spallucci, Un-graviton corrections to the Schwarzschild black hole. Phys. Lett. B 693, 155 (2010). https://doi.org/10.1016/j.physletb.2010.07.058

18. M. Jamil, D. Momeni, M.A. Rashid, Notes on dark energy interacting with dark matter and unparticle in loop quantum cosmology. Eur. Phys. J. C 71, 1711 (2011). https://doi.org/10.1140/epjc/ s10052-011-1711-5

19. R.A. de Souza, J.E. Horvath, Reducing the parameter space for Unparticle-inspired models using white dwarf masses. Phys. Rev. D 86, 027502 (2012). https://doi.org/10.1103/PhysRevD.86.027502

20. G. Alencar, C.R. Muniz, Thermodynamic properties of static and rotating unparticle black holes. JCAP 1803(03), 040 (2018). https:// doi.org/10.1088/1475-7516/2018/03/040

21. F. Sannino, R. Zwicky, Unparticle and Higgs as composites. Phys. Rev. D 79, 015016 (2009). https://doi.org/10.1103/PhysRevD.79. 015016 
22. A. Karch, K. Limtragool, P.W. Phillips, Unparticles and anomalous dimensions in the cuprates. JHEP 1603, 175 (2016). https://doi.org/ 10.1007/JHEP03(2016) 175

23. A.M. Frassino, P. Nicolini, O. Panella, Unparticle Casimir effect. Phys. Lett. B 772, 675 (2017). https://doi.org/10.1016/j.physletb. 2017.07.029

24. H.B.G. Casimir, On the attraction between two perfectly conducting plates. Indag. Math. 10, 261 (1948)

25. H.B.G. Casimir, Kon. Ned. Akad. Wetensch. Proc. 51, 793 (1948)

26. H.B.G. Casimir, Front. Phys. 65, 342 (1987)

27. H.B.G. Casimir, Kon. Ned. Akad. Wetensch. Proc. 100N3-4, 61 (1997)

28. M. Bordag, G.L. Klimchitskaya, U. Mohideen, V.M. Mostepanenko, Advances in the Casimir effect. Int. Ser. Monogr. Phys. 145, 1 (2009)

29. V .B. Bezerra, H .F. Mota, C .R. Muniz, Casimir effect in the rainbow Einstein's universe. EPL 120(1), 10005 (2017). https:// doi.org/10.1209/0295-5075/120/10005

30. V .B. Bezerra, H .F. Mota, C .R. Muniz, Remarks on a gravitational analogue of the Casimir effect. Int. J. Mod. Phys. D 25(09), 1641018 (2016). https://doi.org/10.1142/S0218271816410182

31. V.B. Bezerra, H.R. Christiansen, M.S. Cunha, C.R. Muniz, M.O. Tahim, Thermal Casimir effect in Kerr spacetime with quintessence and massive gravitons. Eur. Phys. J. C 77(11), 787 (2017). https:// doi.org/10.1140/epjc/s10052-017-5378-4

32. C.R. Muniz, V.B. Bezerra, M.S. Cunha, Horava-Lifshitz gravity effects on Casimir energy in weak field approximation and infrared regime. Phys. Rev. D 88, 104035 (2013). https://doi.org/10.1103/ PhysRevD.88.104035

33. G.L. Klimchitskaya, V.M. Mostepanenko, Constraints on axionlike particles and non-Newtonian gravity from measuring the difference of Casimir forces. Phys. Rev. D 95(12), 123013 (2017)
34. M. Blasone, G. Lambiase, L. Petruzziello, A. Stabile, Casimir effect in Post-Newtonian Gravity with Lorentz-violation. Eur. Phys. J. C 78(11), 976 (2018). https://doi.org/10.1140/epjc/ s10052-018-6464-y. [arXiv:1808.04425 [hep-th]]

35. L. Buoninfante, G. Lambiase, L. Petruzziello, A. Stabile, Casimir effect in quadratic theories of gravity. Eur. Phys. J. C 79(1), 41 (2019). https://doi.org/10.1140/epjc/s10052-019-6574-1. [arXiv:1811.12261 [gr-qc]]

36. F. Sorge, Casimir energy in Kerr space-time. Phys. Rev. D 90(8), 084050 (2014). https://doi.org/10.1103/PhysRevD.90.084050

37. R.B. Mann, Black holes of negative mass. Class. Quant. Grav. 14, 2927 (1997). https://doi.org/10.1088/0264-9381/14/10/018

38. J.A. Wheeler, On the Nature of quantum geometrodynamics. Ann. Phys. 2, 604 (1957). https://doi.org/10.1016/ 0003-4916(57)90050-7

39. B. Grinstein, K.A. Intriligator, I.Z. Rothstein, Comments on Unparticles. Phys. Lett. B 662, 367 (2008). https://doi.org/10.1016/j. physletb.2008.03.020

40. V.V. Kiselev, Quintessence and black holes. Class. Quant. Grav. 20, 1187 (2003). https://doi.org/10.1088/0264-9381/20/6/310

41. S.G. Ghosh, Rotating black hole and quintessence. Eur. Phys. J. C 76(4), 222 (2016). https://doi.org/10.1140/epjc/ s10052-016-4051-7

42. B. Toshmatov, Z. StuchlÃk, B. Ahmedov, Rotating black hole solutions with quintessential energy. Eur. Phys. J. Plus 132(2), 98 (2017). https://doi.org/10.1140/epjp/i2017-11373-4

43. V.B. Bezerra, M.S. Cunha, L.F.F. Freitas, C.R. Muniz, M.O. Tahim, Casimir Effect in the Kerr Spacetime with Quintessence. Mod. Phys. Lett. A 32(01), 1750005 (2016). https://doi.org/10.1142/ S0217732317500055 